\title{
Asymmetric collaboration in virtual reality
}

- A taxonomy of asymmetric interfaces for collaborative immersive learning

\section{Lui Albæk Thomsen}

Ph.d.-stipendiat

Department of Architecture, Design, and Media Technology, AAU-CPH

\section{Rolf Nordahl}

\section{Associate Professor}

Department of Architecture, Design, and Media Technology, AAU-CPH

\section{Niels C. Nilsson}

\section{Assistant Professor}

Department of Architecture, Design, and Media Technology, AAU-CPH

\section{Boris Lohmann}

Release Manager \& Projektleder

IT, EduLab ApS.
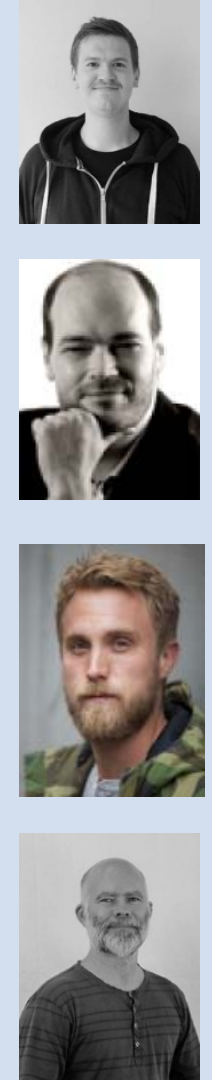


\section{Abstract}

It has been established that Virtual Reality (VR) possess unique qualities for educational purposes. These include the ability to situate the learner at the location or in the perspective that the desired knowledge exists. Within a situation, virtual simulation of systems and mechanism can be used to interact and perform sequences of actions that trains the user in a given task.

In this paper, asymmetrical immersive VR in educational settings will be reviewed, as it is relevant for learning situations where multiple students use the technology together. As an example, in mathematics, asymmetric VR could be used in contexts where unknown variables must be found in collaboration. The purpose of the narrative literature review is to gain greater understanding of how asymmetric game mechanics has influence on communication and collaboration between learners. To map the dynamics of this type of learning activity, a taxonomy will be presented. 


\section{Introduction}

As we are moving towards the maturing of Virtual Reality (VR) technology, new design configurations are being investigated to overcome limitations for multiuser purposes. A new genre of VR applications, containing elements of asymmetry between the users, has recently gained attraction.

The popular asymmetric VR game Keep Talking and Nobody Explodes ${ }^{1}$ has been a great success since being published on digital distribution platforms for video games and VR applications. In short, procedurally generated bombs must be detonated by the immersed player with the help from the non-immersed player equipped with a defusal manual. Various game modes within the genre has been coined by the community with categories such as Asymmetrical Local Multiplayer, Hotseat Local Multiplayer, VR Multiplayer, Online/Cross-platform VR. This genre has also been referred to as Party VR, possibly due to its design conveniently involving multiple people in a partly shared experience. Apart from entertainment, asymmetric VR may possess beneficial elements evoking communication in learning activities where several students collaborate to reach a common goal.

However, the concept of collaborating in Virtual Environments (VEs) is not new to the industrial and scientific communities. Previously, the topic has been defined as the research area of shared workspaces, where distinctions between cooperation, collaboration and teleoperation are made. To draw parallels to existing theory and previous state of the art, the analytical parts of the article will take initial starting point of investigation in this area. In such a scenario, the premise for working together is often symmetric, meaning that each participant has similar system abilities in solving a problem or obtaining an understanding of a subject through critical thinking. Thereafter, we can provide reflections upon the processes' relevance for collaboration between participants with asymmetric immersive interfaces.

The remainder of the paper is structured by the chapters Related Work and Taxonomy. Section [2.1] details the processes that collaborating partners undergo and the associated success criteria to ensure a certain level of quality in the collaboration. The section also details the scenarios and attributes of shared workspaces. Section [2.2] details an approach to designing the virtual experience with focus on game elements contributing to the experience. Section [2.3] presents the degrees of asymmetry defined by the level of interactivity. Section [2.4] details the typology of didactic approaches to learning in VR and the affordances identified in previous

\footnotetext{
${ }^{1}$ Keep Talking and Nobody Explodes: http://www.keeptalkinggame.com/
} 
research. Section [3] presents a taxonomy of design components between interface and participants resulting in communication dynamics and collaboration mechanics.

\section{Related Work}

The following section will present theory on shared workspaces. The shared workspaces of teleoperation, cooperation and collaboration will be described. This is done to provide context for the various scenarios in which a shared workspace can be beneficial. The relevant context for our purpose (i.e., collaboration between students in a classroom setting) will be reflected upon further in the later sections detailing the taxonomy.

\subsection{Collaboration}

The following section will present work on collaboration ranging from traditional human-to-human collaboration to computer-supported collaboration. Finally, a recent study on the use of immersive technology in the context of teleoperation will be described. This context is relevant to our context of immersive collaboration, as the communication situations and processes are similar.

Collaboration is the process of two or more individuals working together to solve a common problem or achieve a common goal. In the process, the quality of the collaboration is often determined by the amount of shared information, knowledge of group and individual activity and coordination of task activities (Dourish \& Bellotti, 1992). At the end of the section, evaluation on the quality of the collaboration will be detailed. According to Gutwin \& Greenberg (2000), the process is divided into seven collaboration mechanics that contribute to the quality of the collaboration (Gutwin \& Greenberg, 2000):

1. Explicit communication: intentional sharing of information (verbal/written), workspace and artifacts contains the information, deictic references ("this one") and gestures (e.g., pointing and gaze).

2. Consequential communication: two types; information given off by artifacts as they are manipulated by others (also known as feedthrough) and information given off by the characteristics of the actions of a person's embodiment in the workspace.

3. Coordination of action: organizing actions to avoid conflicting actions, shared resource/tools require turns to be taken and perhaps ordering of actions. 
4. Planning: low-level planning activities such as dividing a procedure into sequences of tasks, indicating solution to task before it is executed, organising artifacts for the next purpose.

5. Monitoring: workspace awareness information; who, where what (incl. progress according to coordination/planning).

6. Assistance: provide help; opportunistic and informal or explicitly requested.

7. Protection: surveillance of negative influence on work by other persons in the shared workspace (monitoring assists in updating the status on need of protection).

These mechanics are fundamental to understanding the process of asymmetric collaboration with VR. In traditional collaboration, these mechanics are often utilized verbally in conversational formality. In VR, there are possibilities to incorporate automated processes that enables the user to convey and initiate activities digitally through synchronized devices.

According to Gutwin \& Greenberg, evaluation of a collaboration is conducted by assessing three success criteria (Gutwin \& Greenberg, 2000):

- Effectiveness: considers whether the activity was successfully completed (incl. number and severity of errors).

- Efficiency: considers the resources spent (e.g., time or effort).

- Satisfaction: collaborators' satisfaction with the execution (directly influenced by effectiveness and efficiency).

Tullis \& Albert describes similar criteria for measuring the quality of the user experience, which can be done by analysing the following five metrics (Tullis \& Albert, 2008):

1. Task success: the users' experience of completing the task (complete/partial success or failure)

2. Time-on-task: the time elapsed between the start and end of a task (minutes or seconds)

3. Errors: possible outcomes caused by a usability issue

4. Efficiency: combination of time-on-task and required effort of the user

5. Learnability: the extent of which something can be learned efficiently (see also learning curve)

Regarding the full span of the experience, there are also methods from presence research to evaluate the user's experience and behaviour during game play. These will be elaborated upon in section [2.4.] on Immersive Learning. 


\section{Computer-Supported}

The following section will briefly describe the processes of ComputerSupported Cooperative Work (CSCW) and Computer-Supported Collaborative Learning (CSCL), how they differ and where they usually take place.

In cooperative work, a task is split into a procedure containing sequences of activities and each participant is responsible for a portion of the problem solving according to the division of labour (Hamalainen, 2006). In such a setting, each participant is therefore responsible for producing separate solutions that fit in the overall progress of the task execution. The participants in cooperative work is often professionals in a work organization with open or closed skillsets facilitating the required group performance to complete the task (Hamalainen, 2006).

In collaborative learning, the participants are equally involved in a shared activity and must coordinate their cognitive and physical efforts to construct a shared solution (Hamalainen, 2006). The collaborators are instructed about activities and goals by an expert (i.e., the teacher) of the educational institution. However, once the frame of the activity is defined, the students of the educational institution are expected to actively interact with their collaborators to share observations and construct meaning behind the solution (Hamalainen, 2006).

The differences between CSCW and CSCL are summed up in the table below:

\begin{tabular}{l|l|l} 
& \multicolumn{1}{|c|}{$\begin{array}{c}\text { Computer-Supported } \\
\text { Cooperative Work (CSCW) }\end{array}$} & $\begin{array}{c}\text { Computer-Supported } \\
\text { Collaborative Learning (CSCL) }\end{array}$ \\
\hline Setting & Work organizations & Educational institution \\
\hline Activity & $\begin{array}{l}\text { Subtasks with individual } \\
\text { responsibilities }\end{array}$ & $\begin{array}{l}\text { Shared activities on solving } \\
\text { problem }\end{array}$ \\
\hline Goal & Separate solutions & Shared solution \\
\hline Interplay & Professionals (equal) & Teacher/student (expert/novice) \\
\hline & Facilitate group performance & $\begin{array}{l}\text { Natural interaction between team } \\
\text { members }\end{array}$
\end{tabular}

According to Strijbos \& Martens (2001), there are three overall dimensions that define whether a group-based learning activity exists in the cooperative extreme or collaborative extreme (Strijbos \& Martens, 2001). The three dimensions include the following: 
1. Pre-structuring: Pre-structuring presents the frame in which the collaboration should take place - this includes material covering task delegation, communication protocols, and instructional aid (Strijbos \& Martens, 2001).

2. Skills: Skills can vary as being fixed for the specific knowledge that the procedure requires (i.e., closed skills) or more abstract management skills for the actual execution of the procedure (i.e., argumentation, negotiation, and conflict resolution) (Strijbos \& Martens, 2001).

3. Task structuring: The type of the task is related to the last dimension, where the structure of the task and number of solutions contribute to the activity being cooperative or collaborative. The illustration below shows the dimensions contributing to the type of group-based learning activities (Strijbos \& Martens, 2001).

Moving from collaboration supported by computers, the next step describes technology that does more than assisting the participants in the process. Instead of dealing with shared workspaces, we can now address actual Virtual Environments (VEs) for immersive collaboration.

\section{Immersive Teleoperation}

In an article from 2017, a classification on human-to-human communication during the use of immersive teleoperation interfaces was presented (Kraus \& Kibsgaard, 2017). Teleoperation is defined as human users operating machines (e.g., robots) at a distance. The classification derives seven dimensions from two overall categories in teleoperation (i.e., communication situation and communication processes). The five dimensions to communication situations include:

- Copresence: a social sensation that the collaborating human users can experience, depending on the degree of mediation in the system (e.g., single-user, collaborative teleoperation, joint teleoperation) (Kraus \& Kibsgaard, 2017).

- Virtuality: a term borrowed from Paul Milgram's theory on the RealityVirtuality Continuum (Milgram \& Kishino, 1994). The term describes the span from real environments to virtual environments with the intermediates of remote environments and recorded environments (Kraus \& Kibsgaard, 2017).

- Transport: a distinction can be made between so-called low-transport and high-transport situations. In low-transport situations, a virtual or remote object or person appear in the immediate environment, whereas in high-transport situations, presence and copresence is experienced by the virtual or remote environment (Kraus \& Kibsgaard, 2017). 
- Point-of-View: describes the perspective of which the operator is perceiving the experience. A distinction is made between perceiving the experience through first- or third-person perspective. This is closely related to the sensation of virtual body-ownership, which occurs when the view is first-person and a virtual character's movements are mapped to the tracked body movements of the user (Kraus \& Kibsgaard, 2017).

- Asynchronicity: describes the timing of communication being proactive, synchronous, or reactive. Most common social experiences in VR involves synchronous communication, however, for collaborative purposes, there exists qualities in the timing of real-time and recorded events (Kraus \& Kibsgaard, 2017).

The article also presents a classification of the channels in which the communication is flowing. According to the authors, there are four communication channels (Kraus \& Kibsgaard, 2017):

- Auditory without using media (e.g., speech or non-verbal utterances)

- Visual without media (e.g., facial expressions, gaze direction, hand gestures, or full-body gestures)

- Using media (e.g., written text, visuals, audio-visual recordings)

- Others (e.g., haptic, olfactory, etc.)

These four channels can have varying levels of mediation. This distinction is focused on the awareness of the users, and whether the mediation of the communication is explicit, transparent or non-existent (Kraus \& Kibsgaard, 2017).

\subsection{Game Design Framework}

The idea is to address game design principles in the taxonomic discussion, since it represents choices from the designer that ultimately has impact on the experience (of the collaboration). The primary purpose of the paper is to describe the possible hierarchical pathways from technology to human-tohuman collaboration. However, it should not be undermined how the designer defines the certain path. Design choices about interaction mechanics, dependence between players and rewarding or progression systems, are what defines the perceived and objective quality of the collaboration. Research has shown that adding game elements to educational activities is a complicated matter, however, it generally supports the hypothesis that it can foster motivation and performance in the participants (Sailer, Hense, Mayr, \& Mandl, 2017). The influence of specific game design elements in asymmetric immersive collaboration is prone to further research, which will be summed up by the end of the paper. Before 
commencing into the specifications of asymmetry in immersive experiences for collaboration, some basic principles about game design will be established. The following theoretical principles will take basis in the Mechanics, Dynamics and Aesthetics (MDA) framework (Hunicke, Leblanc, \& Zubek, 2005) (see Figure 1).

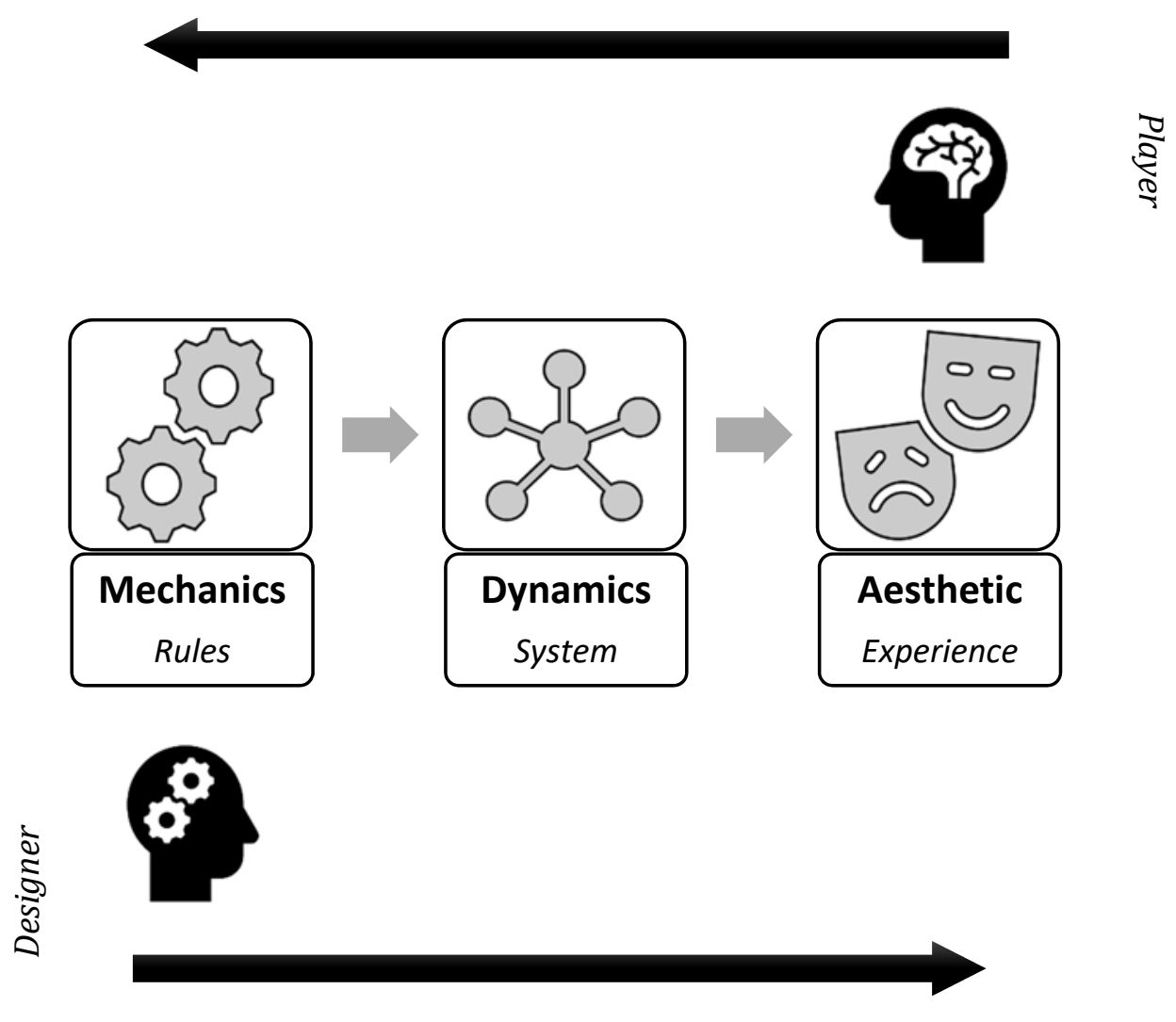

Figure 1: The MDA Framework. Figure adapted from (Hunicke, Leblanc, \& Zubek, 2005).

Mechanics describes the components of the game, at the level of data representation and algorithms (e.g., "the rules of the game"). Dynamics describes the run-time behaviour of the mechanics acting on player inputs and each other's outputs over time (e.g., "how players use the rules"). Aesthetics describes the desirable emotional responses evoked in the player, when the person interacts with the game system (e.g., "how the game makes the player feel'). There are two different perspectives on the final product: a) the game designer explicitly knows the inner mechanism of the game but needs to understand the perceived aesthetics from the player, b) the player experiences the aesthetics of the game but needs to understand the mechanics to proceed in the required skills of the game. 


\section{Mechanics}

As mentioned previously, mechanics of a game constitutes the "inner" components of the game contributing to the dynamic experience. The experience will now be put into the perspective of asymmetric gameplay. According to Harris et al. (2016), there are six mechanical manipulations that designers can employ in order to give rise to asymmetric gameplay experiences (Hunicke, Leblanc, \& Zubek, 2005).

- Ability - virtual abilities are different between players

- Challenge - virtual challenges are different between players

- Interface - physical interfaces are different between players

- Information - virtual or physical access to information is different between players

- Investment - the dedicated time of virtual roles is different between players

- Goal/Responsibility - the goal of the game is different between players

Regarding asymmetric VR, there is a natural asymmetry in the mechanic of Interface. The first player is immersed using a head-mounted display (HMD) with stereoscopic vision. This allows the user to perceive a threedimensional virtual environment with depth cues. The second player (or group of players) can either be equipped with instructions on paper or digital screen. The input modalities of each group vary quite a lot as well. The user with immersive equipment can have spatially tracked input devices with 3 or 6 Degrees of Freedom (DoF). The non-immersed users are limited to screen-based gestures (e.g., smartphone or tablet), discrete or continuous inputs (e.g., keyboard or mouse) or simply non-interactive interfaces (e.g., a document).

\section{Limitation}

Given that the paper will not address specific game design elements influence on collaboration, the asymmetric mechanics will be narrowed down to three core mechanics determined to be essential in establishing a collaborative environment. As previously mentioned, the goal/responsibility in CSCW is often shared by participants, and for now, this mechanic will be disregarded with the intention of further investigation in the future perhaps a clear distinction between goal and responsibility needs to be made. Instead of addressing the asymmetric mechanics of investment and challenge, the related mechanic of abilities and information will be represented instead. 


\section{Dynamics}

Defining the dynamics of the experience, the two participating groups of the experience will be labelled for the sake of readability. Depending on the didactic approach and curricular design, there may be more than one person in the non-immersed group, however, the idea remains that copresence is not necessarily present, as one person is perceiving the workspace through VR and the rest are perceptually located in the real environment. These two groups can be divided into actor and assistant(s). In a collaborative process, the interdependence between participants is crucial to the dynamics experienced at run-time. The actor must coordinate with the assistant in asymmetric VR and contribute to the problem-solving or critical thinking where they are required in order to meet the shared goal of the collaboration (Harris, Hancock, \& Scott, 2016). This relationship is described by the directional dependence and the synchronicity between participants.

The directional dependence between participants in a learning activity with asymmetric interfaces is defined by the didactic approach and curricular design. Harris et al. presents three directions of dependence; mirrored, unidirectional and bidirectional. These directions describe reliance relationship between participants and the three configurations can be seen in Figure 2.

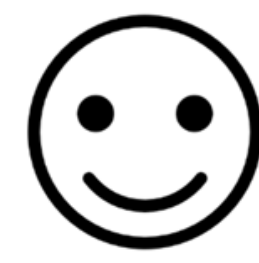

Actor

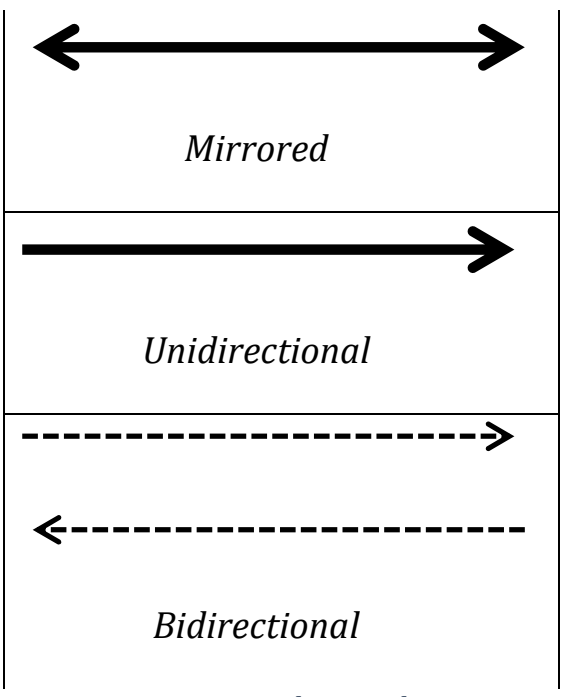

Figure 2: Directional Dependencies

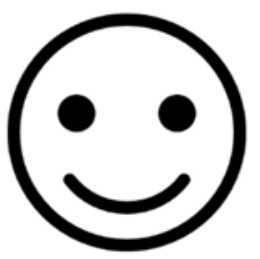

Assistant(s)

The possibilities of directional dependence for our purpose is discussed in section [3.3] according to the three levels of asymmetry. 
Following the description on directional dependence, it is appropriate to outline the timing of events between participants. There can be varying durations of activities and the start- and endpoints are crucial to the coordination of the collaboration. Each participant's actions within the collaboration can be distinguished between discrete events or continuous. This distinction is relevant both to hardware inputs and user inputs in collaborative learning with asymmetric VR. Later in the paper, the possibilities of input devices in such a system will be presented. Harris et al. presents five unique combinations of events timing (Harris, Hancock, \& Scott, 2016):

- Asynchronous Timing occurs when Player A performs an action (either discrete or continuous) and Player $B$ is concerned with the specifics of when.

- Sequential (Disjoint) Timing occurs when Player A completes their action some time $(\Delta t)$ before Player $B$ begins their action

- Expectant Timing occurs when Player A can trigger an action if Player $B$ is prepared (and waiting)

- Concurrent Timing occurs when both Player A and Player B continuously perform their respective actions.

- Coincident Timing occurs when Player A and Player B must perform discrete actions at the same moment (or within some small $\varepsilon$ ).

The possibilities of synchronicity and timing for our purpose is discussed later according to the three levels of asymmetry.

\section{Aesthetics}

In the MDA framework, the component of aesthetics aims to provide more objective descriptions to the profile of the game constituted by mechanics and dynamics. To avoid subjective vocabulary such as "fun", "exciting", and "entertaining", the component contains the following taxonomy of descriptors; sensation (sense-pleasure), fantasy (make-believe), narrative (drama), challenge (obstacle course), fellowship (social framework), discovery (uncharted territory), expression (self-discovery) and submission (past-time) (Harris, Hancock, \& Scott, 2016). A game can incorporate multiple of these descriptors, and ultimately, it is the interplay between components that influences the perceived aesthetics of the game.

Aesthetics for our purpose is highly influenced by characteristics of output displays. These include visual, auditory and haptic displays. In the concluding parts of the paper, the possibilities and associated characteristics are mapped out. 


\subsection{Asymmetry}

There can be said to be different degrees of asymmetry in the setup. Depending on the game and collaboration mechanics, there can be said to be three degrees of asymmetry in the interfaces of a collaborative learning activity in virtual environments. Depending on the didactic approach and curricular design, there may be more than one person in the non-immersed group, however, the idea remains that one person is perceiving the workspace through a head-mounted display. These two groups can be divided into actor and assistant.

The variable that influences the degree of asymmetry is the interactivity of the assistant. We distinguish between three levels of asymmetry; low, medium and high (see Figure 3). These three levels are defined by the abilities provided by the interaction; direct (e.g., view direction or manipulative actions), indirect (e.g., 2D coordinate, buttons, text, voice) or none (e.g., verbal communication).
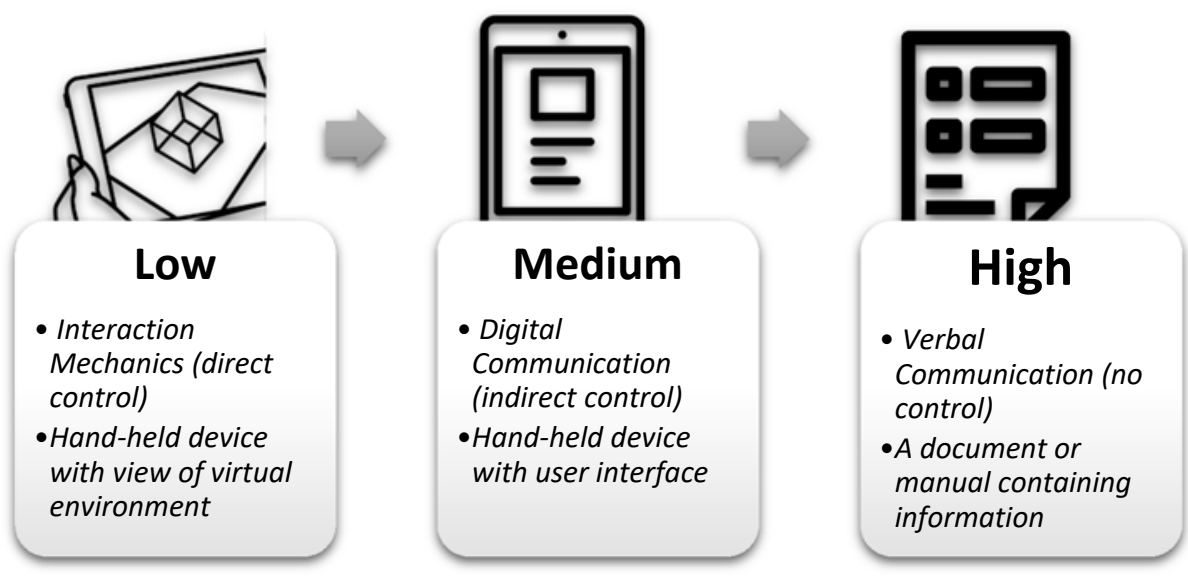

Figure 3: Degrees of Interaction Asymmetry for Assistant in Asymmetric Collaboration

Low asymmetry is present when the assistant is capable of viewing and directly influencing the environment (e.g., changing perspective or manipulate objects). Medium asymmetry is present when the assistant can transfer information digitally through an interconnected digital interface. 
High asymmetry is present when the assistant's abilities are purely analogue, and information must be conveyed verbally.

\subsection{Immersive Learning}

Immersive learning is the experience of acquiring knowledge in an augmented or virtual environment, which can be transferred to the realworld (known as knowledge transfer). Oftentimes, the experience is perceived through a head-mounted display or a digital screen (working as a "window") into the virtual environment. The range of immersive technologies are defined in the Reality-Virtuality Continuum and includes media such as Virtual Reality (VR) and Augmented Reality (AR) (Milgram \& Kishino, 1994).

The educational quality that immersive media possesses has preliminarily been identified to be its ability to situate and immerse the learner in the place or perspective in which the desired knowledge exists (referred to as situated learning) (Liu, Dede, Huang, \& Richards, 2017). Immersive media has also been found to enrich curricula material, promote active forms of learning, measure performance of high validity, as well as provide an opportunity for academic learning in familiar situations (Liu, Dede, Huang, \& Richards, 2017). In mathematics (and geometry), three key affordances have been identified to be the interactive manipulation and construction of three-dimensional geometry, practicing spatial relationships and the reification of abstract problems (Kaufmann, 2009).

In recent times, as part of the Digital Identity, Curation and Education (DICE) research network, the project of VR School Research ${ }^{2}$ has been established. The ongoing project investigates how immersive VR can be used to enhance learning, its relationship to curriculum and its implications for pedagogy. The project has led to a number of reports and publications on learning affordances ${ }^{3}$, safety protocols (Southgate, et al., 2018), teacher guidelines ${ }^{4}$ and literature review (Southgate, Immersive Virtual Reality, children and school education: A literature review for teachers, 2018).

As part of the scientific assessment of the learning affordances for the virtual lab simulations company Labster, a study was recently published questioning the effects of using VR. The purpose of the study was to determine the consequences of adding immersive VR to virtual learning

\footnotetext{
2 VR School Research: https://vrschoolresearch.com/

${ }^{3}$ The Power of Virtual Reality for Education by Erica Southgate:

https://ericasouthgateonline.files.wordpress.com/2018/08/infographic-learning-affordances-of-vr-a4.pdf

${ }^{4}$ Top tips for teachers on the learning affordances of virtual reality by Erica Southgate:

https://ericasouthgateonline.files.wordpress.com/2018/08/top-tips-for-teachers-on-the-learning-affordances-of-vr.pdf
} 
simulations, and to investigate if the principles of multimedia learning are applicable to immersive VR (Makransky, Terkildsen, \& Mayer, 2017). The findings of the study include that although it does possess motivating properties, learning science in VR may overload and distract the learner, resulting in less opportunity to build learning outcomes (Makransky, Terkildsen, \& Mayer, 2017).

Another study from 2017 reviews the use of virtual reality head-mounted displays in education and training (Jensen \& Konradsen, 2018). Bibliometric analysis was performed on 21 documents to assess quality and analyse the studies' quality. According to the Medical Education Research Study Quality Instrument, the overall score was below average and especially for usercentred evaluations for product-oriented designs. However, skills acquisition in VR was identified to be useful in several studies (e.g., cognitive skills, psychomotor skills, and affective skills). In any other situations, the authors describe the experience to be counterproductive, due to widespread cybersickness, technological challenges and - similarly to the findings of Makransky et al.'s study - overloading and distracting the learning from building learning outcomes (Jensen \& Konradsen, 2018).

Several Danish studies have also been conducted on learning in VR for various purposes. Majgaard \& Lyk (2015) concludes that constructivism (as well as experience-based learning) as a didactic approach in visual arts education can help students obtain better understanding of the technology and associated creative design processes (Majgaard \& Lyk, 2015). In a report on learning with spherical videos (also known as 360-degree cinematography), it is concluded that students prefer spherical video as a good alternative to traditional electronic educational technology incorporating regular video material to teach physiotherapy (Ulrich, et al., 2016). Recently, a paper has been published on teaching mathematics with VR technology. It is concluded that in the experimental design, aesthetic learning processes were enabled by the technology and experienced by the students resulting in an increase of motivation associated with the learning activity (Hansen \& Jensen, 2018).

\section{Didactic Approaches}

Immersive educational applications can be designed by a simulating an artefact, environment, situation, or procedure from real life (Liu, Dede, Huang, \& Richards, 2017). There are different didactic approaches that contribute to the engagement of the user in the immersive learning experience. These include embodied cognition, directed immersive narrative, and constructionist activities. 
Immersive learning experiences benefit from embedded cognition, which naturally occurs when a VR-system establishes an illusion of non-mediation of immersive virtual environments. This phenomenon is also referred to as the place-illusion and can be enhanced by sensations of virtual bodyownership and interaction mechanics (Slater, 2009). An embodied immersive experience can develop a useful mental perceptual simulation useful when retrieving a concept or reasoning about it, especially when facilitated by curricular and instructional support (Liu, Dede, Huang, \& Richards, 2017). As an example, the student is immersed into a remote and/or inaccessible location. The reasons why the location might be inaccessible can be due to history, budget plans and safety. Such an experience resembles a traditional local expedition trip of a school class (e.g., a trip to the zoo or historical place). The children are taken on a trip to the location, in which the knowledge is perceivable from. The experience is usually limited to changing perspective through head orientation in terms of interaction. With newer technologies in volumetric capturing and photogrammetry, the experience might become fully interactive with 6DoF and photorealistic artefacts and environments. Examples of software from the state of the art in situated learning can be seen below, where users are teleported to historical or presently inaccessible locations.

Learning through life-like simulations was first established with flight simulators going all the way back to 1929 (see Edwin A. Link's flight trainer) and research has since then proven its validity (Oritz, 1993). Today's consumer market for desktop PCs and the advancement of game engines allows the programmer to incorporate and customize interactive real-time simulations in the virtual environment (such as PhysX by Nvidia ${ }^{5}$ ). Examples of software from the state of the art in simulation training include applications such as Labster $V R^{6}$ and Calcflow $V R^{7}$, where users can learn through biology and safety in laboratory simulations or interact with mathematical models and graphs in a three-dimensional coordinate system.

Constructionist learning theory assumes that developing knowledge occurs best through building artefacts (physical or virtual) that can be experienced and shared. In this type of learning, participants are given tools to build their own immersive environments or inhabit the environment with selfconstructed virtual artefacts. This approach can be very effective, because it empowers the learners to create something in which they have an emotional

\footnotetext{
${ }^{5}$ Nvidia GeForce PhysX: https://www.geforce.com/hardware/technology/physx

${ }^{6}$ Labster VR on Daydream: https://www.labster.com/vr/

${ }^{7}$ CalcFlow: https://nanome.ai/calcflow/
} 
investment, while promoting higher-order thinking skills such as problemsolving and critical thinking (Liu, Dede, Huang, \& Richards, 2017).

The experiences can be directed by letting the learners participate in shaping a narrative. Apart from being guided through the instructional design, they also have the possibility to choose own paths from reasoning and critically assessing options in the immersive experience (Liu, Dede, Huang, \& Richards, 2017).

For simpler material, such as math facts or vocabulary words, the experience is not perceived as intrinsically interesting and students seem to suffer from fatigue of covering up the simple material with artistically expressive extrinsic rewards (e.g., music, animations, mini-games) (Liu, Dede, Huang, \& Richards, 2017).

\section{Taxonomy}

In this section, we will present a taxonomy on system components that may facilitate collaboration using asymmetric immersive interfaces between an actor and assistant(s). The taxonomy is based on the previously presented research on collaboration mechanics, communication dynamics, game design, and asymmetric game mechanics. In order to extend the taxonomy to address the effects of asymmetric layouts on learning, it is required that empirical data is gathered from students testing the suggested system configurations. This will be conducted in future work of the author group.

In immersive learning, the tasks and information of the VEs is limited by the characteristics of the input and output devices in the system. Before a designer takes decision on the dynamics of the game, the abilities and information of the experience is defined by the hardware capabilities. Therefore, the preliminary taxonomy takes starting point in the interface, which is the main asymmetric component of the collaborative learning experience with asymmetric immersive interfaces we are describing. The additional mechanics have been deemed to primarily rely on the design choices and intended experience by the designers, and these will therefore not be included in the current taxonomy. There is a need to conduct more research and evaluate case studies on the tuning of factors contributing to the collaboration. There is also a need for further technical documentation of libraries and tools to implementing system features allowing constructive and usable digital collaboration mechanics and communication dynamics. Initially, the overall taxonomy and mapping between components can be seen below (see Figure 4). Examples of asymmetric setups with regards to the previous presented degree of interaction asymmetry will be illustrated 
later using the taxonomy. Components are color-coded by the category they belong to and dependency between each are shown by the connected links.

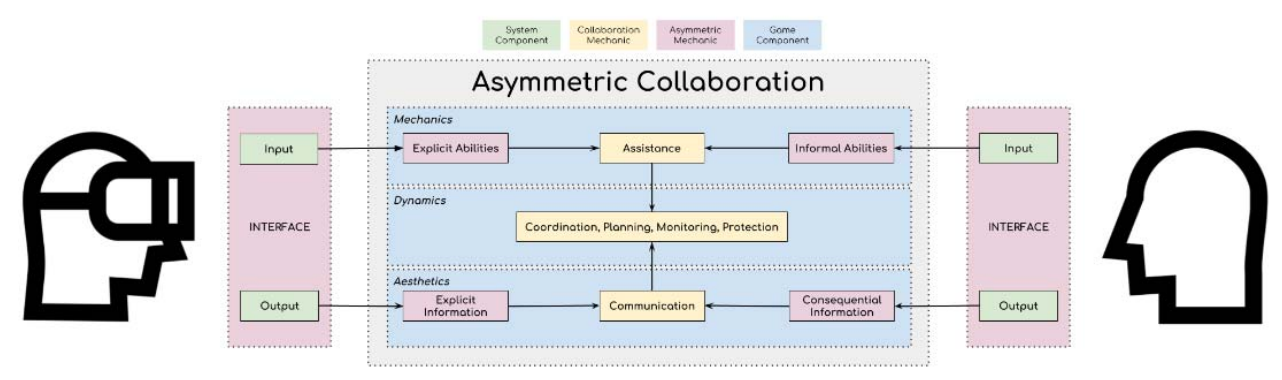

Figure 4: Taxonomy on Collaboration with Asymmetric Immersive Interface

Before commencing into the details of individual components and frameworks of the taxonomy, an outline and short description of these can be presented:

- Asymmetric mechanics

- Hardware components

- Game components

- Collaboration mechanics

Asymmetric mechanics were described in section [2.3.1.] and for our purpose, a selection of the mechanics was included. Hardware components are essential to the investigation, as they are fundamental to an immersive experience and establishes the foundation for communicating dynamic content inside of the immersive environment. Game components are based on the MDA framework and constitutes perspectives of the designer and the player of the game. Collaboration mechanics were described in [2.1.] and consists of seven mechanics. In order to provide context for the taxonomy, two examples containing high and low levels of asymmetry has been illustrated. The examples describe two scenarios in which the interface of the non-VR assistant is different. In the first example, we see high asymmetry between the two users, given that the actor has full access and information about a virtual 3D environment, while the assistant is handed a document or manual containing instructions. This case is similar to the game design of Keep Talking and Nobody Explodes, which was briefly covered in the introduction of the article. 


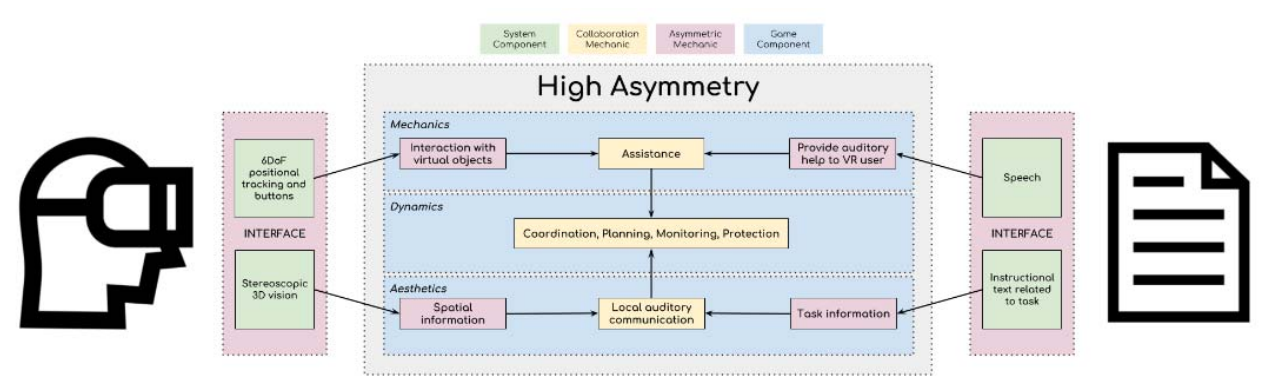

Figure 5: Taxonomy on Collaboration with Asymmetric Immersive Interface

In the second example, the two users' perception of the workspace is almost shared. The assistant has the ability make observations on the environment from a mobile "window" to the virtual environment but does not possess the same abilities to manipulate the environment as the main actor does. However, the informative inputs of the assistant may seem more intuitive to main actor, as they arise from perceiving the environment (although without the equal level of immersion).

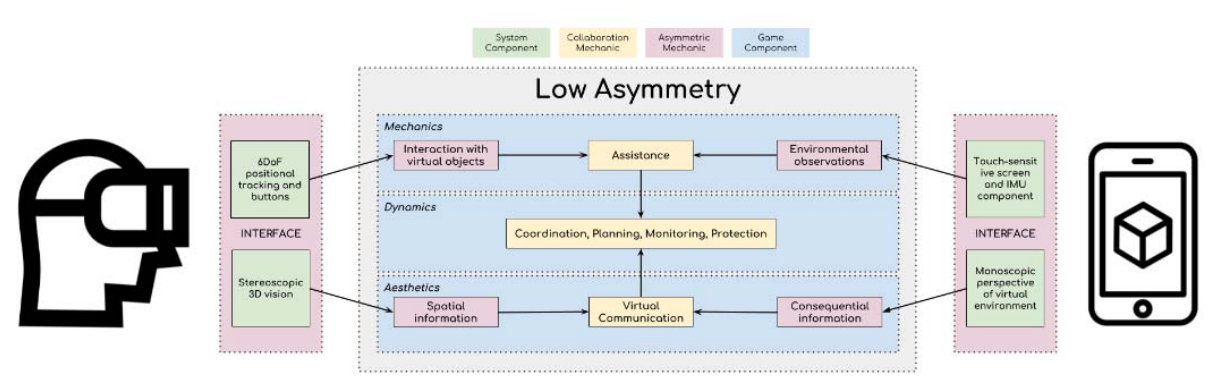

Figure 6: Taxonomy on Collaboration with Asymmetric Immersive Interface

\subsection{Collaboration Mechanics}

The components of asymmetric mechanics in the taxonomy were narrowed down to three from the initial five as defined in the literature. The purpose of the taxonomy is to describe the inner components between hardware and users, which enable dynamics contributing to collaborative learning and, therefore, the mechanics of Challenge, Investment and Goal/Responsibility were found to be at a higher level in the design being based on the didactic approach, curricular design and game design.

\subsection{Hardware Components}

Hardware components take basis in the distinction between input and output devices. For a clearer overview in the main taxonomy, the variations of the input and output devices are presented in an additional diagram. 
Currently, the configuration for assistant in the collaborative learning system can be done in two ways. The assistant can be provided with direct, indirect or non-interactive instructions. Recalling the section of Asymmetry, these mechanics result in varying levels of asymmetry. The non-interactive instructions can be provided on a document or in the form of a passive application on a mobile device. The application may take similar shape to the document, simply providing information in an organized manner. The application can also be made interactive to either provide indirect inputs to the system (e.g., 2D coordinate, buttons, text, voice) or directly influence the game play for the immersed user (e.g., view direction or manipulative actions). In the next subsection, the input capabilities of both participants (immersed actor and non-immersed assistants) are mapped out as User Inputs. For the output capabilities of the system, a classification of types of information has been included to indicate the content of the displays for the assistant in the collaborative setup. Both diagrams are color-coded by the following: blue represents features that are available to the immersed actor and pink represents the features available to the non-immersed assistant(s).

\section{User Inputs}

The mechanics of each group is defined by the ability to directly or indirectly influence the collaboration (see Figure 7). Direct input is provided inside the IVE and can be categorized into the following tasks; selection, manipulation, navigation, and system control (LaViola, Kruijff, McMahan, Bowman, \& Poupyrev, 2017). Indirect input to the system can only happen if the assistant is provided with an interactive interface. These inputs include changing the perspective, 2D coordinate position data, button inputs, and text or voice inputs. These do not directly influence the state of the IVE, however, they provide inputs that may assist the immersed actor in the task.

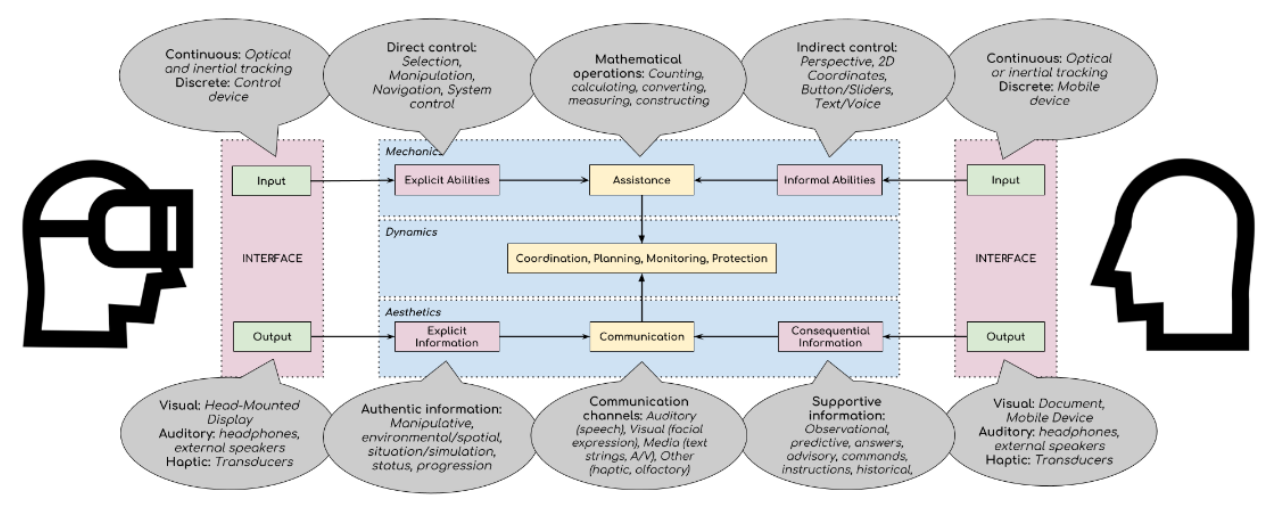

Figure 7: User Inputs 


\section{Hardware Inputs}

Initially, the devices providing inputs to the system can be divided into analogue and discrete data (see Figure 8). Analogue devices provide the system with continuous data instead of binary data. This can be exemplified by tracking versus button inputs. Oftentimes, tracking systems consists of complex sensor fusion to calculate the position and orientation of a plane in 3D space. This data is numerical as opposed to discrete data, which in the context of games is often labelled in a binary manner (e.g., on or off). Discrete data is therefore often seen as input from control devices (e.g., hand-held controllers). However, hand-held controller is often compromised by multiple input data types. For example, regular buttons provide discrete data to the system, but tracking pads or joysticks can provide numerical data for the current position in a 2D coordinate system. A mobile device can simulate both numerical and discrete data through the design of the user interface. Additionally, in almost all cases of smartphones, the mobile device also has embedded inertial measurement units (IMU) providing information about orientation through sensor fusion between angular-rate gyroscopes, linear accelerometers and magnetometers (LaViola, Kruifff, McMahan, Bowman, \& Poupyrev, 2017). These components have varying degree of vividness and the associated characteristics are depicted at the end of the diagram.

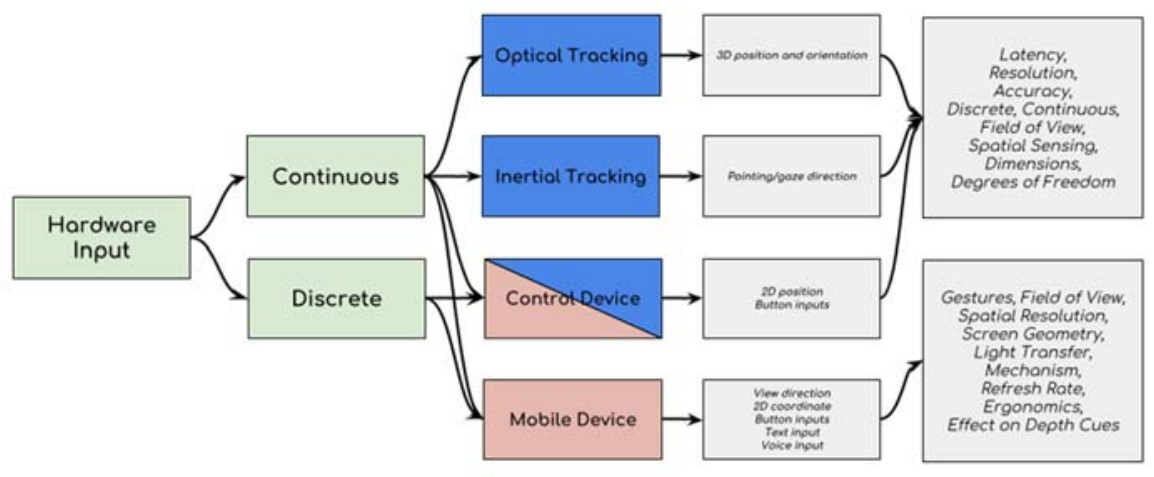

Figure 8: Hardware Inputs

\section{Output}

In the domain of output, there are three display categories that constitute the multisensory experience; visual, auditory and haptic (LaViola, Kruijff, McMahan, Bowman, \& Poupyrev, 2017). The immersed actor is often stimulated with the combination of the three displays with immersion inducing qualities (see characteristics in Figure 9), however, for the nonimmersed assistant(s), the visual display is non-immersive with varying 
degrees of interactivity. As previously mentioned, information of the display for the non-immersed assistant(s) is based on a classification of information for visual displays (Buck, 1983).

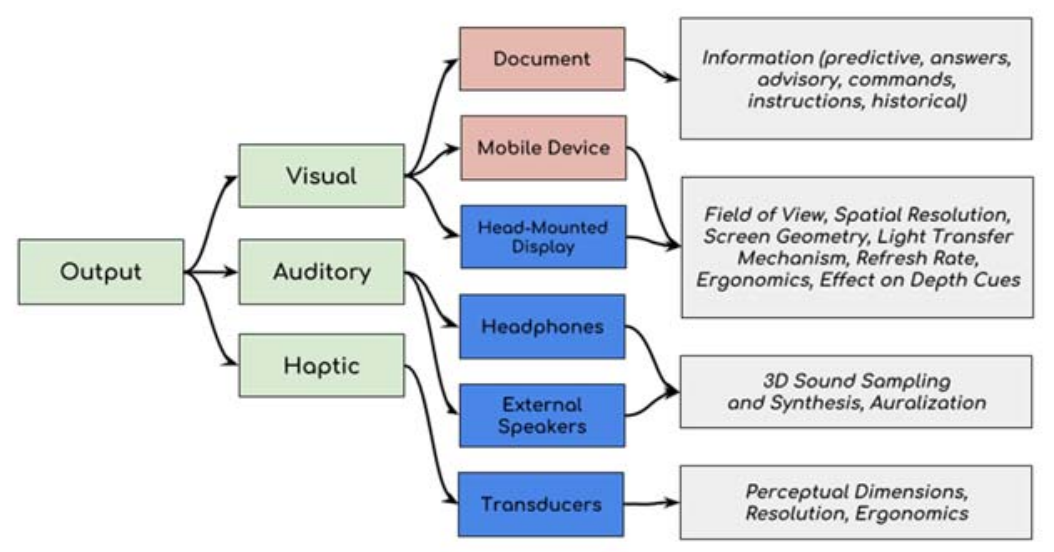

Figure 9: System Output

\subsection{Communication Dynamics}

The incorporated components related to the structure of the game is based on the MDA framework, as previously described. Mechanics in this context are treated as the abilities provided to the user by the physical and virtual interface. It is important to distinguish between these two domains, because in the virtual domain, motions can be mapped to any imaginable outcome. The dynamics of the communication are highly influenced by the channels in which the communication is conveyed. Based on classification of communication channels (Kraus \& Kibsgaard, 2017), four types are mapped out as part of the taxonomy between communication and coordination. We argue that the MDA framework is influential to the communication as the components are defining to what (e.g., observation) and when (e.g., manipulation) communication is possible in the system. As previously mentioned, it is important for future work to document the effects and design of the communication channels (e.g., processing and analysis of information, data representation, etc.).

\section{Dependence \& Synchronicity}

The dynamics of the collaboration is influenced by the dependency and timing of events. We argue that when similar interaction mechanics (e.g., full-body interaction of the actor and direct interaction of the assistant), the dependency is mirrored to the extent that both participants can manipulate similar content of the collaboration (see figure 9). With medium asymmetry 
(e.g., full-body interaction of the actor and indirect interaction of the assistant), there is a unidirectional dependency, since both participants are dependent on each other, but in different ways (e.g., manipulate object or convey information about problem solution). Finally, in high asymmetry, the actor is exclusively dependent on the assistant conveyance of information. The assistant does not have any access to changing the outcome of performing tasks in the $\mathrm{VE}$, and therefore, only the dynamics of the communication is present.

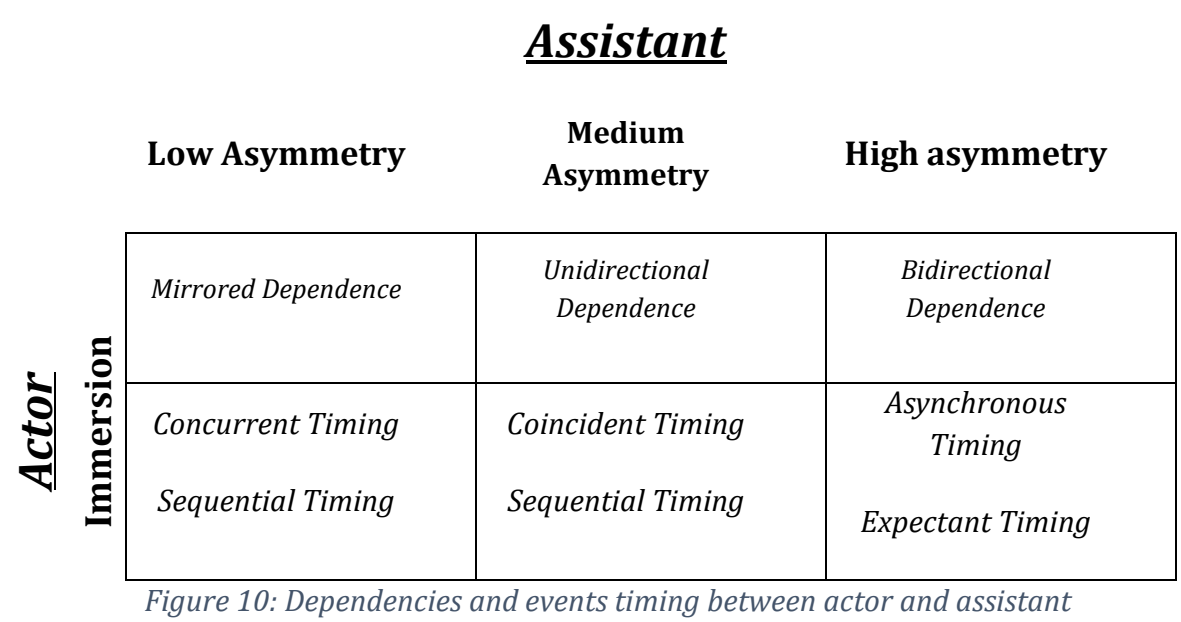

Based on the definitions on synchronicity (Harris, Hancock, \& Scott, 2016), a preliminary mapping of types of event timing is presented above (see Figure 10). The argument here is that the distinction between actions and concerns. Shared action dependency moves towards lower asymmetry and exclusive actions and individual concerns moves towards higher asymmetry. In both concurrent and sequential timing, it is insinuated that both participants have influence in the tasks of the VE. However, it can also be argued that semantics decide the positioning on the scale, and therefore, overlapping can exist. Coincident timing suggests that the main concern is discrete actions, which are possible in both low and medium asymmetric configurations, however, the assistant is more likely to have discrete actions as main interaction in setups with medium asymmetry. Finally, asynchronous timing and expectant timing suggests that the main actor performs actions that the assistant needs to keep timing of or be prepared to act upon, which do not necessarily require any levels of interactivity. This subsection describes the thought process of organizing dependency and events timing between different layouts of asymmetry, however, it is required that the preliminary suggestions are more thoroughly investigated with user studies and technical documentations. 


\section{Conclusion}

The paper presents the concept of collaborative learning with asymmetric immersive interfaces. Investigation of previous techniques for shared workspaces and group-based learning has been presented to draw parallels between the two concepts of collaborating in digital and virtual environments. Didactic approaches to immersive learning and game design frameworks have been included to investigate the scenario in which children are learning through collaboration with asymmetric immersive interfaces. Finally, a taxonomy and clarifying sub-diagrams have been presented in the previous section to map the components contributing to collaboration between interface and participants. The paper is part of a larger industrial research project and future work includes verifying or falsifying the statements and connections presented. As mentioned throughout the paper, there are several factors' effects on learning outcome and collaboration quality that needs to be investigated in future work:

- Level of asymmetry, immersion and multimodality

- Asymmetric mechanics (incl. investment, challenge, and goal/responsibility)

- Didactic approaches and reflection on curricular designs

- Communication channels

- Collaboration mechanics

- Specific game design elements

In future work, the listing above will be formulated into hypotheses for the purpose of testing with Danish primary schools' students in classroom settings. It is also important that we address an important failure of the concept; asymmetric learning outcome. Designing guidelines on how to ensure equal challenge between actors and assistants should aid educators in understanding the process of designing valuable immersive experiences for multiple users with limited number of head-mounted displays. There are many theoretical concerns presented in the paper, however, the reality of schools is different and administrative and organizational limitations often weight the most in defining the experiences. 


\section{References}

Buck, J. R. (1983). Visual Displays. Human Factors: Understanding PeopleSystem Relationships, s. 195-231.

Dourish, P., \& Bellotti, V. (1992). Awareness and Coordination in Shared Workspaces. CSCW' 92 Proceedings of the 1992 ACM conference on Computer-supported cooperative work, s. 107-114.

Gutwin, C., \& Greenberg, S. (2000). The Mechanics of Collaboration: Developing Low Cost Usability Evaluation Mehods for Shared Workspaces. Proceedings IEEE 9th International Workshops on Enabling Technologies: Infrastructure for Collaborative Enterprises (WET ICE). doi:10.1109/ENABL.2000.883711

Hamalainen, R. (2006). Designing and evaluatong collaboration in a virtual game environment for vocational learning. Computers \& Education, s. 98-109.

Hansen, C., \& Jensen, M. E. (2018). Astetiske læreprocesser med virtual reality i matematik. Lærerprofession.dk.

Harris, J., Hancock, M., \& Scott, S. D. (19. October 2016). Leveraging Asymmetries in Multiplayer Games: Investigating Design Elements of Intedependent Play. CHI Play '16 Proceedings of the 2016 Annual Symposium on Computer-Human Interaction in Play, s. 350-361. doi:10.1145/2967934.2968113

Hunicke, R., Leblanc, M., \& Zubek, R. (2005). MDA: A Formal Approach to Game Design and Game Research. I Technology and the Lifeword. University Press. Hentet fra http://www.cs.northwestern.edu/ hunicke/MDA.pdf

Jensen, L., \& Konradsen, F. (2018). A review of the use of virtual reality head-mounted displays in education and training. Education and Information Technologies, s. 1515-1529.

Kaufmann, H. (2009). Virtual Environments for Mathematics and Geometry Education. Themes in Science and Technology Educatio, vol. 2(131152). Hentet fra http://earthlab.uoi.gr/theste/index.php/theste/article/view/60 
Kraus, M., \& Kibsgaard, M. (2017). A Classification of Human-to-Human Communication during the Use of Immersive Teleoperation Interfaces. Journal of Virtual Reality and Broadcasting(1).

LaViola, J. J., Kruijff, E., McMahan, R. P., Bowman, D., \& Poupyrev, I. P. (2017). 3D User Interfaces: Theory and Practice (2nd Edition udg.). Addison-Wesley Professional.

Liu, D., Dede, C., Huang, R., \& Richards, J. (2017). Virtual, Augmented, and Mixed Realities in Education. Springer. doi:10.1007/978-981-105490-7

Majgaard, G., \& Lyk, P. (2015). På Rejse med Virtual Reality i billedkunst: erfaringslæring gennem kombineret fysisk og virtual modelbygning. Læring \& Medier(14).

Makransky, G., Terkildsen, T. S., \& Mayer, R. E. (2017). Adding immersive virtual reality to a science lab simulation causes more presence but less learning. Learning and Instruction.

Milgram, P., \& Kishino, F. (1994). A Taxonomy of Mixed Reality Visual Displays.

Oritz, G. A. (1993). Transfer of Learning Effectiveness: PC-Based Flight Simulation. Journal of Aviation/Aerospace Education \& Research, s. 8.

Sailer, M., Hense, J. U., Mayr, S. K., \& Mandl, H. (2017). How gamification motivates: An experimental study of the effects of specific game design elements on psychological need satisfaction. Compters in Human Behavior, s. 371-380.

doi:https://doi.org/10.1016/j.chb.2016.12.033

Slater, M. (2009). Place Illusion and Plausibility can lead to realistic behaviour in immersive virtual environments. Philisophical Transactions of the Royal Society B: Biological Sciences, s. 35493557.

Southgate, E. (2018). Immersive Virtual Reality, children and school education: A literature review for teachers. DICE Report Series Number 6. Newcastle: DICE Research. Hentet fra http://dice.newcastle.edu.au/DRS_6_2018.pdf

Southgate, E., Smith, P. S., Eather, G., Saxby, S., Cividino, C., Bergin, C., ... Kilham, J. (2018). Ethical Conduct and Student Safety in Immersive 
Virtual Reality: Protocols and Resources from the VR School Research Project. IEEE VR (Workshop on K-12+ Embodied Learning through Virtual \& Augmented Reality (KELVAR).

Strijbos, J. W., \& Martens, R. L. (2001). Group-based Learning: Dynamic interaction in groups. Proceedings of EURO-CSCL Conference, s. 569576.

Tullis, T., \& Albert, W. (2008). Measuring the User Experience: Collecting, Analyzing and Presenting Usability Metrics. Elsevier.

Ulrich, F., Helms, N. H., Frandsen, U., Rafn, A. V., Vestbo, M., \& Jungfalk, M. (2016). E-Læring og Virtual Reality: et kontrolleret eksperiment med e-læring og sfærisk VR ved de sundhedsfaglige professionsuddannelser. UCSJ. 